\title{
Elevated international normalised ratios correlate with severity of injury and outcome
}

\author{
M A Noorbhai, ${ }^{1} \mathrm{MB}$ BCh, FCS (SA), MMed (Surg); H M Cassimjee, ${ }^{2} \mathrm{MB}$ ChB, DA (SA), FCS (SA), Cert Crit Care (SA), Cert Trauma Surg (SA); \\ B Sartorius, ${ }^{3} \mathrm{PhD}$; D J J Muckart, ${ }^{4} \mathrm{MB}$ ChB, FRCS, Cert Crit Care (SA), MMedSci \\ ${ }^{1}$ Department of General Surgery, School of Clinical Medicine, College of Health Sciences, Nelson R Mandela School of Medicine, University of \\ KwaZulu-Natal, Durban, South Africa \\ ${ }^{2}$ Trauma Unit and Trauma Intensive Care, Inkosi Albert Luthuli Central Hospital, Durban, South Africa \\ ${ }^{3}$ Discipline of Public Health Medicine, School of Nursing and Public Health, College of Health Sciences, Nelson R Mandela School of Medicine, \\ University of KwaZulu-Natal, Durban, South Africa \\ ${ }^{4}$ Trauma Unit and Trauma Intensive Care, Inkosi Albert Luthuli Central Hospital, Durban, South Africa
}

Corresponding author: M A Noorbhai (noorbhai@ukzn.ac.za)

Background. Haemorrhagic shock is the leading cause of preventable early deaths from trauma. Acute coagulopathy on admission to a trauma unit is associated with worse outcomes. The relationship of haemorrhage to early mortality remains consistent regardless of mechanism of injury. Haemorrhage and haemorrhagic shock are increasingly amenable to interventions that result in reductions in morbidity and mortality.

Objectives. To assess the prevalence of coagulopathy in patients admitted to the level 1 trauma unit at Inkosi Albert Luthuli Central Hospital, Durban, South Africa, and correlate it with in-hospital mortality.

Methods. A retrospective analysis of the first 1000 patients admitted to the trauma unit during the years 2007 - 2011 was performed. The admission international normalised ratios (INRs) were correlated with Injury Severity Scores (ISSs) and in-hospital mortality. A multivariable Poisson model with robust standard errors was used to assess the relationship between coagulopathy and mortality after adjustment for the confounding influence of age and gender. The data were analysed using the $R$ statistics program.

Results. Of the 1000 patients, 752 were male. There were 261 admissions directly from the scene and 739 inter-hospital transfers (nonscene). The mean INRs among survivors for all, scene and non-scene patients were 1.33, 1.30 and 1.34, respectively, and those among non-survivors $1.92,2.01$ and 1.88 , respectively $(p<0.001)$. The overall prevalence of coagulopathy was $48.7 \%, 46.9 \%$ in scene patients and $49.2 \%$ in non-scene patients. The mortality rate of scene patients with abnormal INR levels was $41.1 \%$ (adjusted relative risk (aRR) $3.59,95 \%$ confidence interval (CI) $2.11-6.44 ; p<0.001)$ v. $25.1 \%$ for non-scene patients (aRR 1.67, 95\% CI $1.15-2.05 ; p=0.004)(p=0.001$ ).

Conclusions. There was a high prevalence of coagulopathy in our study. Raised admission INRs were associated with worse outcomes. There was a direct correlation between the INR and the ISS. INRs may offer predictive capabilities in resource-depleted environments where the ISS is not routinely calculated. Early recognition of acute coagulopathy may help reduce morbidity and mortality.

S Afr Med J 2016;106(11):1141-1145. DOI:10.7196/SAMJ.2016.v106i11.10356

Eighty percent of deaths from trauma occur within the first 24 hours after injury. ${ }^{[1]}$ Central nervous system (CNS) injuries and haemorrhagic shock are the leading causes of early mortality. ${ }^{[2]}$ However, their respective prevalence may vary depending on trauma service and reporting. Despite advances in trauma care, uncontrolled haemorrhage is responsible for $>50 \%$ of trauma-related deaths ${ }^{[2]}$ and is the most common cause of preventable deaths. ${ }^{[3]}$

The significance of haemorrhage control cannot be overemphasised, as in addition to directly causing death, $25 \%$ of CNS injuries are complicated by shock, increasing CNS mortality twoto three-fold. ${ }^{[4-6]}$ This relationship to early mortality ascribed to CNS injuries and haemorrhage remains consistent regardless of mechanism of injury. ${ }^{[2,7]}$ Despite advances in trauma care, there are still few interventions that can directly improve outcome from severe primary CNS trauma. ${ }^{[8]}$

Haemorrhagic shock, however, is the leading cause of early potentially preventable trauma deaths, and recognition of the acute coagulopathy of traumatic shock (ACoTS) has made this increasingly amenable to interventions that result in reductions in morbidity and mortality. ${ }^{[2,9-13]}$ In association with major haemorrhage, acute coagulopathy on admission is reported to be associated with worse outcomes. ${ }^{[14]}$

\section{Objectives}

To assess the prevalence of ACoTS at the level 1 trauma unit at Inkosi Albert Luthuli Central Hospital (IALCH), Durban, South Africa (SA), and correlate it with injury severity and in-hospital mortality.

\section{Methods \\ Study design}

The study was approved by the local ethics review board (Biomedical Research Ethics Committee, University of KwaZulu-Natal, Durban, ref. no. BE131/09).

\section{Study setting}

The study was conducted in the level 1 trauma unit at IALCH during the years 2007 - 2011. The trauma unit receives patients either directly from the scene of the incident or via inter-hospital transfers. Patients' hospital numbers were retrieved from the existing trauma unit database and used to access each individual patient record from the computerised hospital information system. We used Soarian (Siemens, Germany), which allows real-time data entry including patient demographics, clinical notes, and serological and radiological investigations. The first 1000 patients admitted to the trauma unit were included in the study. The data were de-identified prior to assessment. 
The variables extracted included clinical parameters, mechanism of injury, INR, coagulation profile and in-hospital mortality. All blood results were those taken on admission to the resuscitation room via a direct-puncture arterial sample in a non-heparinised syringe. INR results were extracted from the electronic hospital laboratory database. The INR was analysed at the hospital laboratory using a Siemens BCS XP System (Siemens, Germany). ${ }^{[15]}$ Innovin (DadeBehring, Germany) was the thromboplastin used. An INR $>1.2$ was considered indicative of coagulopathy, in accordance with previous studies. ${ }^{[16,17]}$

Missing blood results in the first hour of admission were excluded from the study. The mechanism of injury was obtained from the patient's electronic chart. The Injury Severity Score (ISS) ${ }^{[18]}$ was computed after all injuries had been identified using the Abbreviated Injury Scale 90 reference book. ${ }^{[19]}$ Death or survival was ascertained from the electronic hospital discharge notes.

The data were processed and analysed using the $R$ statistics program (R Core Team, 2014). ${ }^{[20]}$ INRs were recorded and analysed in 939 patients. The INR was dichotomised into $\leq 1.2$ and $>1.2$. These were then correlated with ISS and in-hospital mortality. The bivariate analysis employed Pearson's $\chi^{2}$ test to determine the association between INR category and various outcomes/predictors, e.g. mortality. This analysis was then further stratified by scene/nonscene. Furthermore, a multivariable Poisson regression with robust standard errors was performed to assess the relationship between coagulopathy and mortality after adjustment for age and gender. Coefficients were exponentiated to represent adjusted relative risks (aRRs). A $p$-value of $<0.05$ was considered statistically significant.

\section{Results}

\section{Demographics}

The first 1000 patients were included in the study, of whom 752 were male (Table 1). The average age was 29 years (median 27 , interquartile range (IQR) 21 - 36, standard deviation (SD) 15). The majority of admissions were from the 21 - 30-year age group (36.9\%), with 1.6\% aged $>70$ years and $16.5 \%<16$ years.

\section{Scene v. non-scene}

There were 261 patients (26.1\%) admitted directly from scene, the remaining 739 (73.9\%) being inter-hospital transfers (non-scene).

\section{INR in all admissions}

INRs were not recorded in 61 patients, who were excluded from the analysis (Table 2). Of the remaining 939 patients, $454(48.3 \%)$ were not found to have coagulopathy, while 485 (51.7\%) had INRs $>1.20$. The overall mean INR was 1.46 (SD 1.77), (median 1.20, IQR 1.09 - 1.40). Survivors had significantly lower INRs (mean 1.33, median 1.18, IQR 1.07 - 1.33) than non-survivors (mean 1.92, median 1.35, IQR 1.14 - 1.68) $(p<0.001)$.

The overall mortality rate was $21.9 \%$, the rate being $15.4 \%$ in patients with a normal INR and $28.9 \%$ in those with a raised INR $(p<0.001)$. Patients with an INR $>1.20$ were approximately two times more likely to die than those with a normal INR (adjusted RR 2.05, 95\% CI 1.60 - 2.63) (Table 3). This is higher than the crude estimate as a result of negative confounding from age, i.e. patients with coagulopathy were significantly younger than their counterparts, and older individuals were more likely to die. There was a trend of

Table 1. Characteristics of the patients in the study

\begin{tabular}{|c|c|c|c|c|c|}
\hline & \multirow[b]{2}{*}{ All patients } & \multicolumn{2}{|c|}{ Scene patients } & \multicolumn{2}{|c|}{ Non-scene patients } \\
\hline & & $\begin{array}{l}\text { No coagulopathy } \\
\text { (INR } \leq 1.20 \text { ) }\end{array}$ & $\begin{array}{l}\text { Coagulopathy } \\
(\text { INR }>1.20)\end{array}$ & $\begin{array}{l}\text { No coagulopathy } \\
\text { (INR } \leq 1.20 \text { ) }\end{array}$ & $\begin{array}{l}\text { Coagulopathy } \\
(\mathrm{INR}>1.20)\end{array}$ \\
\hline Patients, $n$ & 1000 & $121 / 228$ & $107 / 228$ & $361 / 711$ & $350 / 711$ \\
\hline Age (years), mean (SD) & $29(15)$ & $34(15)$ & $28(16)$ & $31(15)$ & $27(15)$ \\
\hline Males, $n(\%)$ & $752(75.2)$ & $78(64.5)$ & $74(69.2)$ & $271(75.1)$ & $275(78.6)$ \\
\hline INR, mean (SD) & $1.46(1.77)$ & $1.09(0.07)$ & $1.93(2.61)$ & $1.08(0.08)$ & $1.83(2.44)$ \\
\hline Coagulopathy prevalence, $\%$ & 48.7 & - & 46.9 & - & 49.2 \\
\hline Mortality, \% & 21.9 & 12.4 & 41.1 & 16.3 & 25.1 \\
\hline \multicolumn{6}{|l|}{ ISS, median (SD) } \\
\hline All patients & $25(16)$ & - & - & - & - \\
\hline Scene & - & $18(14)$ & $29(20)$ & - & - \\
\hline Non-scene & - & - & - & $22(13)$ & $25(15)$ \\
\hline
\end{tabular}

Table 2. Incidence of coagulopathy and mortality for scene and non-scene patients according to ISS

\begin{tabular}{|c|c|c|c|c|c|c|c|c|c|c|}
\hline & \multicolumn{2}{|c|}{ ISS 0 - 15} & \multicolumn{2}{|c|}{ ISS 16 - 30} & \multicolumn{2}{|c|}{ ISS 31 - 45} & \multicolumn{2}{|c|}{ ISS 46 - 60} & \multicolumn{2}{|c|}{ ISS $61-75$} \\
\hline & Norm. & Coag. & Norm. & Coag. & Norm. & Coag. & Norm. & Coag. & Norm. & Coag. \\
\hline \multicolumn{11}{|l|}{ Scene $(N=228)$} \\
\hline$n$ & 45 & 14 & 48 & 42 & 20 & 27 & 4 & 14 & 2 & 12 \\
\hline Died, $n(\%)$ & $1(2.2)$ & $1(7.1)$ & $6(12.5)$ & $9(21.4)$ & $4(20.0)$ & $12(44.4)$ & $3(75.0)$ & $12(85.7)$ & $1(50.0)$ & $10(83.3)$ \\
\hline \multicolumn{11}{|c|}{ Non-scene $(N=711)$} \\
\hline$n$ & 43 & 73 & 163 & 162 & 98 & 76 & 44 & 29 & 13 & 10 \\
\hline Died, $n(\%)$ & $5(11.6)$ & $14(19.2)$ & $21(12.9)$ & $29(17.9)$ & $12(12.2)$ & $27(35.5)$ & $14(31.8)$ & $12(41.4)$ & $6(46.2)$ & $7(70.0)$ \\
\hline
\end{tabular}


increasing deaths for each increase in INR interval (Fig. 1). The only INR interval with $0 \%$ mortality was $0.80-0.89$, suggesting that even within the so-called normal limits of the INR $(0.8$ - 1.2) patients are still vulnerable to adverse outcomes.

\section{INR in scene patients}

INRs were not recorded in 33 of the 261 patients admitted directly from the scene. Of the remaining 228 patients, 121 (53.1\%) did not have coagulopathy, while 107 (46.9\%) had INRs $>1.20$. Survivors had significantly lower INRs (mean 1.30, median 1.16, IQR 1.06 - 1.29) than non-survivors (mean 2.01, median 1.42, IQR 1.21 - 1.73) ( $p=0.01)$. The overall mortality rate was $25.9 \%$, patients with a normal INR having a $12.4 \%$ mortality rate while the rate in those with a raised INR was $41.1 \%(p<0.001)$. Scene patients with an INR $>1.20$ were approximately four times more likely to die than scene patients with a normal INR (aRR 3.59, 95\% CI 2.12 - 6.06) (Table 3).

\section{INRs in non-scene patients}

There were 739 patients transferred from other hospitals, among whom the INR was not recorded in 28. Of the remaining 711 patients, 361 (50.8\%) had INRs within normal limits and $350(49.2 \%)$ had INRs >1.20. Survivors had significantly lower INRs (mean 1.34, median 1.19, IQR 1.08 - 1.34) than non-survivors (mean 1.88, median 1.30, IQR $1.13-1.64)(p<0.001)$. The overall mortality rate was $20.7 \%$, patients with a normal INR having a $16.3 \%$ mortality rate while the rate in those with a raised INR was $25.1 \%$ ( $p=0.004)$. Non-scene patients with an INR $>1.20$ were $\sim 1.7$ times more likely to die than non-scene patients with a normal INR (aRR 1.67, 95\% CI 1.26- 2.23) (Table 3).

\section{Severity of injury}

The ISS was calculated for each patient. The incidence of coagulopathy increased as the ISS increased. The median overall ISS was 25, being 20 for survivors (IQR 14 - 29) and 34 for non-survivors (IQR 25 - 50). The median ISS in scene patients who survived was 18 (IQR 9 - 28), the score for non-survivors being 50 (IQR 34 - 66). The mean ISS score for non-scene patients who survived was 22 (IQR $16-29)$ and that for non-survivors 30 (IQR 18 - 41).

Among scene patients, the lowest ISSs associated with coagulopathy were $16-30$, with ISSs of $31-45$ in non-scene patients. After adjusting for age and gender, increasing ISS was significantly associated with an increased risk of mortality among all patients as well as when stratified as scene/non-scene

Table 3. Multiple Poisson regression analysis for all, scene and non-scene patients, adjusted for age and male gender

\begin{tabular}{|c|c|c|c|c|c|c|c|c|}
\hline & \multicolumn{4}{|c|}{ INR model } & \multicolumn{4}{|c|}{ ISS model } \\
\hline & Variable & aRR & 95\% CI & $p$-value & Variable & aRR & $95 \% \mathrm{CI}$ & $p$-value \\
\hline \multirow[t]{3}{*}{ All } & $\mathrm{INR}>1.20$ & 1.92 & $1.49-2.48$ & $<0.001$ & ISS & 1.04 & $1.03-1.04$ & $<0.001$ \\
\hline & Age & 1.02 & $1.01-1.03$ & $<0.001$ & Age & 1.02 & $1.01-1.02$ & $<0.001$ \\
\hline & Male & 1.10 & $0.83-1.46$ & 0.516 & Male & 1.21 & $0.96-1.55$ & 0.113 \\
\hline \multirow[t]{3}{*}{ Scene } & INR $>1.20$ & 3.68 & $2.11-6.44$ & $<0.001$ & ISS & 1.04 & $1.03-1.05$ & $<0.001$ \\
\hline & Age & 1.01 & $1.00-1.02$ & 0.017 & Age & 1.01 & $1.00-1.02$ & 0.059 \\
\hline & Male & 0.97 & $0.62-1.50$ & 0.884 & Male & 1.00 & $0.73-1.36$ & 0.978 \\
\hline \multirow[t]{3}{*}{ Non-scene } & INR $>1.20$ & 1.54 & $1.15-2.05$ & 0.004 & ISS & 1.03 & $1.02-1.04$ & $<0.001$ \\
\hline & Age & 1.02 & $1.01-1.03$ & $<0.001$ & Age & 1.02 & $1.01-1.03$ & $<0.001$ \\
\hline & Male & 1.21 & $0.83-1.76$ & 0.316 & Male & 1.40 & $0.98-2.01$ & 0.062 \\
\hline
\end{tabular}

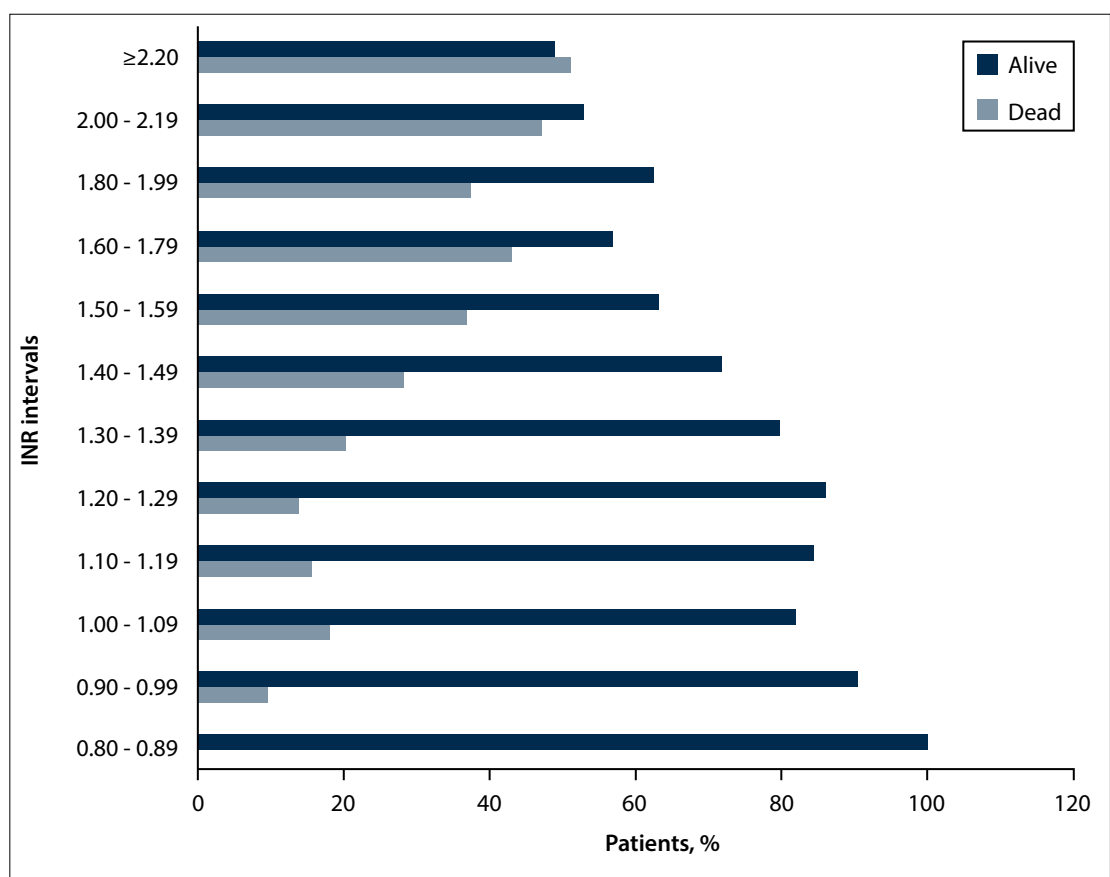

Fig. 1. INR intervals and associated outcomes.

(Table 3). Overall a one-unit increase in ISS after adjustment was associated with an $\sim 4 \%$ increased risk of mortality (aRR 1.04; $p<0.001)$. This effect size was similar when stratified by scene/non-scene.

Among patients who died, there was a greater degree of coagulopathy for every ISS interval for both scene and non-scene patients. Table 2 illustrates the incidence of coagulopathy and mortality in scene and non-scene patients categorised by ISS. The presence of coagulopathy was also significant when deaths in resuscitation, in the operating theatre and within 24 hours of ICU admission were excluded (Fig. 2).

The majority of patients with an INR of $<1.30$ have a higher probability of being alive despite their injury severity. However, the INR interval $1.30-1.49$ shows a pro- gressively increased probability of death as the ISS increases. Patients with an INR $>1.49$ have a high probability of death across all ISS intervals. In this study, the overall prevalence of coagulopathy was $34.1 \%$ for an ISS $<15$, $47.3 \%$ for an ISS of $16-30,57.1 \%$ for an ISS of $31-45$ and $70.1 \%$ for an ISS $>45$. Both the scene and non-scene groups had an increased prevalence with increasing ISS scores.

\section{Discussion}

Invented by Kirkwood ${ }^{[21]}$ in an effort to consistently record prothrombin ratios, the INR is the ratio of patient's prothrombin time (PT) divided by the mean normal PT raised to the power of the International Sensitivity Index (ISI). Before the INR was available, many different prothrombin time 


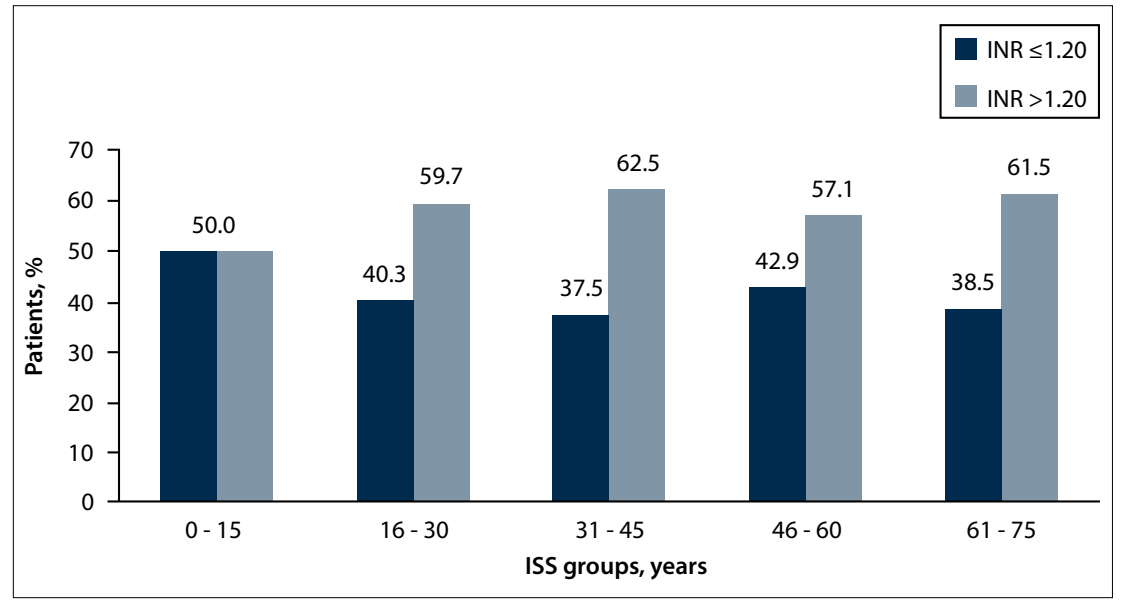

Fig. 2. All mortality according to ISS v. INR levels (deaths in rescuscitation, the operating theatre and early intensive care excluded).

ratios were recorded based on the type of thromboplastin reagent used. Kirkwood ${ }^{[21]}$ also produced the ISI, which allowed the calibration of different thromboplastins to an international standard. The INR is therefore a more precise way than other methods of accurately recording the prothrombin ratios and the degree of coagulation.

The INR and PT are measures of the extrinsic pathway of coagulation. The PT measures the following clotting factors: factor I (fibrinogen), factor II, factor V, factor VII and factor X. The activated partial thromboplastin time (aPTT) measures factors VIII, IX and XII of the intrinsic pathway and factors $\mathrm{V}, \mathrm{X}$, fibrinogen and prothrombin of the common pathway. The INR/PT and aPTT are therefore screening tests for detecting critical clotting factor deficits that impair haemostasis. ${ }^{[22]}$

Rizoli et al. ${ }^{[2]}$ analysed clotting factor levels in patients with severe trauma (ISS 216). They found that patients without a critical clotting factor deficiency all had normal INRs and aPTTs, i.e. that the INR and aPTT had a specificity of $100 \%$ for critical clotting factor deficits. ${ }^{[22]}$ Factor $\mathrm{V}$ was found to be particularly low compared with other clotting factors. ${ }^{[2]}$ They also noted that thromboelastography was inferior to INR or aPTT in identification of critical clotting factor deficits. ${ }^{[23]}$ Critical clotting factor deficiencies occur immediately after injury, before substantial fluid resuscitation, and are associated with poor outcomes. At the time of our study, neither ROTEM products (TEM, Switzerland) nor a point-ofcare (POC) INR device were available in our unit. Our findings suggest that a raised INR is associated with increased mortality rates and that both the INR and the ISS can be used to identify patients at risk.

\section{Incidence of ACoTS}

The prevalence of coagulopathy in our study is higher than the rates reported by Brohi et al. ${ }^{[14]}$ ACoTS is reported in $25 \%$ of trauma patients, with an associated four-fold increase in mortality. ${ }^{[14,24,25]}$ Wafaisade et al. ${ }^{[26]}$ and Maegele et al. ${ }^{[24]}$ reported prevalence rates of coagulopathy at admission of $36.1 \%$ and $34.2 \%$, respectively. Similar rates were seen in combat casualties. ${ }^{[27]}$ In our study, the overall prevalence of coagulopathy was $48.7 \%$, with prevalences of $46.9 \%$ and $49.2 \%$ in scene and non-scene patients, respectively.

We further identified prevalence rates for each subgroup of ISS. There was an overall increased prevalence of coagulopathy as ISSs increased. This was true for both scene and non-scene patients. The mortality rate for patients with coagulopathy in each ISS category was higher than that for patients with no coagulopathy. However, among the non-scene patients the mortality rates for both patients with normal coagulation and those with coagulopathy were similar for each ISS category. This suggests that these patients may benefit from earlier direct referral to a level 1 trauma unit. In resourcedepleted environments and in regional and district hospitals where ISSs are not calculated, INRs may be used to identify high-risk patients.

Our results show that the incidence of coagulopathy was increased with increases in ISS. Increased tissue injury manifested by the ISS is related to a proportional increase in coagulopathy. The ISS risk ratio for the entire study population suggested that as the ISS increases, the risk of dying increases significantly.

\section{Study limitations}

INRs can be deranged in conditions other than trauma, including chronic liver disease, and as a result of anticoagulation therapies. The median age and IQR in our study suggests that these conditions were unlikely. Clotting tests were performed at $37^{\circ} \mathrm{C}$ and not at body temperature. Similar studies report similar limitations. ${ }^{[1,25,28]}$ Further analysis of the non-scene group, including amount and type of fluid administration, could offer further insight into the increased prevalence of coagulopathy in this group.

\section{Recommendations}

While ISSs are also predictive of poor outcomes, these scores are not routinely calculated in regional and district hospitals. The INR may offer predictive capabilities to the attending doctor in resourcedepleted environments. POC devices offer the opportunity to obtain INRs faster than conventional coagulation tests.

\section{Conclusions}

ACoTS is often present in trauma patients, particularly the severely injured. The overall prevalence of coagulopathy was comparable to previous studies and was high in both direct admissions and inter-hospital transfers. Raised admission INRs were associated with worse outcomes. There was a direct correlation between INRs and ISSs. The INR may help identify patients at risk in resource-depleted environments. Further studies will assist in identifying optimal overall cut-off values for INR, ISS and ISS subgroups that would help identify patients at risk. Earlier recognition of ACoTS may help reduce mortality.

Acknowledgements. We thank Mr M Habib Noorbhai and Mrs Shireen Khan for their assistance with data collection and their support.

Author contributions. MAN, through a literature review, analysed the feasibility of the study, designed the study, obtained the necessary approvals, did data management and analysis, data tabulation and data illustration, and wrote the manuscript. HMC provided supervision, guided the study design, literature review and data analysis, and read the final manuscript. BS did the statistical analysis and read the statistical aspects of the final manuscript. DJJM computed the ISSs, provided study guidance and read the final manuscript.

1. Hoyt DB, Bulger EM, Knudson MM, et al. Death in the operating room: An analysis of a multi-center experience. J Trauma Acute Care Surg 1994;37(3):426-432.

2. Sauaia A, Moore FA, Moore EE, et al. Epidemiology of trauma deaths: A reassessment. J Trauma Injury Infect Crit Care 1995;38(2):1851-1893.

3. Maegele M, Schöchl H, Cohen MI. An update on the coagulopathy of trauma. Shock 2014:41(Suppl 1):21-25. DOI:10.1097/SHK.0000000000000088 
4. Chesnut RM, Marshall LF, Klauber MR, et al. The role of secondary brain injury in determining outcome from severe head injury. J Trauma 1993;34(2):216-222.

5. Chesnut R, Marshall S, Piek J, et al Farly and late systemic hypotension as a frequent and fundamental source of cerebral ischemia following severe brain injury in the Traumatic Coma Data Bank Unterberg A, Schneider G-H, La Unterberg A, Schneider G-H, Lanksch W, eds. Monitoring of Cerebral Blood Flow and Metabolism in

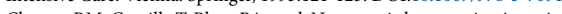

Chesnut RM, Gautille T, Blunt BA, et al. Neurogenic hypotension in patients with severe head injuries. J Trauma Injury Infect Crit Care 1998;44(6):958-963. DOI:10.1097/00005373-199806000-00003

7. Evans JA, van Wessem KJ, McDougall D, et al. Epidemiology of traumatic deaths: Comprehensive population-based assessment. World J Surg 2010;34(1):158-163. DOI:10.1007/s00268-009-0266-

8. Nortje J, Menon DK. Traumatic brain injury: Physiology, mechanisms, and outcome. Curr Opin Neurol 2004;17(6):711-718. DOI:10.1097/00019052-200412000-0001

9. Kauvar DS, Lefering R, Wade CE. Impact of hemorrhage on trauma outcome: An overview of epidemiology, clinical presentations, and therapeutic considerations. J Trauma Injury Infect Crit Care 2006;60(6):S3-S11. DOI:10.1097/01.ta.0000199961.02677.19

10. Gruen RL, Brohi K, Schreiber M, et al. Haemorrhage control in severely injured patients. Lancet 2012;380(9847):1099-1108. DOI:10.1016/S0140-6736(12)61224-0

11. Khan S, Allard S, Weaver A, et al. A major haemorrhage protocol improves the delivery of blood component therapy and reduces waste in trauma massive transfusion. Injury 2013:44(5):587-592. DOI:10.1016/j.injury.2012.09.029

12. Schreiber M. The beginning of the end for damage control surgery. Br J Surg 2012;99(S1):10-11. DOI:10.1002/bjs.7773

13. Borgman MA, Spinella PC, Perkins JG, et al. The ratio of blood products transfused affects mortality in patients receiving massive transfusions at a combat support hospital. J Trauma Injury Infect Crit Care 2007;63(4):805-813. DOI:10.1097/TA.0b013e3181271ba3

14. Brohi K, Singh J, Heron M, et al. Acute traumatic coagulopathy. J Trauma Acute Care Surg 2003;54(6):1127-1130. DOI:10.1097/01.TA.0000069184.82147.06

15. Siemens. BCS XP System 2010-2014. June 2014. http://www.healthcare.siemens.com/hemostasis/ systems/bcs-xp-system (accessed 15 March 2015)

16. Duchesne JC, Islam TM, Stuke L, et al. Hemostatic resuscitation during surgery improves survival in patients with traumatic-induced coagulopathy. J Trauma Injury Infect Crit Care 2009;67(1):33-39. DOI:10.1097/TA.0b013e31819adb8e
17. Gonzalez EA, Moore FA, Holcomb JB, et al. Fresh frozen plasma should be given earlier to patients requiring massive transfusion. J Trauma Injury Infect Crit Care 2007;62(1):112-119. DOI:10.1097/01. ta.0000250497.08101.8b

18. Baker SP, O’Neill B, Haddon W Jr, et al. The Injury Severity Score: A method for describing patients with multiple injuries and evaluating emergency care. J Trauma Acute Care Surg 1974;14(3):187-196. Copes WS, Champion HR, Sacco WJ, et al. Progress in characterizing anatomic injury. J Trauma Injury Infect Crit Care 1990;30(10):1200-1207.

20. R Core Team. R: A Language and Environment for Statistical Computing. Vienna, Austria: R FoundaR Core Team. R: A Language and Environment for Statistical Computing. Vienna, Austrian
tion for Statistical Computing, 2014. http://www.R-project.org (accessed 15 April 2015).

21. Kirkwood TB. Calibration of reference thromboplastins and standardisation of the prothrombin time ratio. Thromb Haemost 1983;49(3):238-244.

22. Rizoli SB, Scarpelini S, Callum J, et al. Clotting factor deficiency in early trauma-associated coagulopathy. J Trauma 2011;71(5 Suppl 1):S427-S434. DOI:10.1097/TA.0b013e318232e5ab

23. Parr MJ, Bouillon B, Brohi K, et al. Traumatic coagulopathy: Where are the good experimental models? J Trauma Injury Infect Crit Care 2008;65(4):766-771. DOI:10.1097/TA.0b013e31818606d2

24. Maegele M, Lefering R, Yucel N, et al. Early coagulopathy in multiple injury: An analysis from Registry on 8724 patients, Injury 2007:38(3):298-304. DOI:10.1016/ injury.2006.10.003

25. MacLeod JB, Lynn M, McKenney MG, et al. Early coagulopathy predicts mortality in trauma. J Trauma Injury Infect Crit Care 2003;55(1):39-44. DOI:10.1097/01.TA.0000075338.21177.EF

6. Wa Wa A multivariate and

27. Niles SE, McLaughlin DF, Perkins JG, et al. Increased mortality associated with the early coagulopathy of trauma in combat casualties. J Trauma Injury Infect Crit Care 2008;64(6):1459-1465. DOI:10.1097 TA.0b013e318174e8bc

28. Floccard B, Rugeri L, Faure A, et al. Early coagulopathy in trauma patients: An on-scene and hospital admission study. Injury 2012;43(1):26-32. DOI:10.1016/j.injury.2010.11.003

Accepted 17 December 2015. 